Cahiers $d u$ MONDE RUSSE

\section{Cahiers du monde russe}

Russie - Empire russe - Union soviétique et États indépendants

$49 / 4 \mid 2008$

Destins individuels et terreur. Jeunesse dans la société post-stalinienne

\title{
Leonid Heretz, Russia on the Eve of Modernity
}

\section{Michel Tissier}

\section{(2) OpenEdition}

Journals

Édition électronique

URL : https://journals.openedition.org/monderusse/6926

DOI : 10.4000/monderusse.6926

ISSN : $1777-5388$

Éditeur

Éditions de l'EHESS

Édition imprimée

Date de publication : 28 décembre 2008

Pagination : 724-727

ISBN : 978-2-7132-2197-2

ISSN : $1252-6576$

Référence électronique

Michel Tissier, "Leonid Heretz, Russia on the Eve of Modernity », Cahiers du monde russe [En ligne], 49/4 | 2008, mis en ligne le 23 décembre 2009, consulté le 03 septembre 2022. URL : http://

journals.openedition.org/monderusse/6926; DOI : https://doi.org/10.4000/monderusse.6926

Ce document a été généré automatiquement le 3 septembre 2022.

Tous droits réservés 


\title{
Leonid Heretz, Russia on the Eve of Modernity
}

\author{
Michel Tissier
}

\section{RÉFÉRENCE}

Leonid HERETZ, Russia on the Eve of Modernity. Popular Religion and Traditional

Culture under the Last Tsars. Cambridge : Cambridge University Press, 2008, X-268 p.

1 Le livre de Leonid Heretz part d'une mise en cause de l'historiographie dominante dans les études russes sur la fin de la période tsariste. Il reproche aux historiens, à la suite des observateurs occidentalisés de l'époque, d'avoir négligé d'étudier la « vision du monde traditionnelle de la paysannerie russe» (p.7), et de ne pas avoir pris en compte les conséquences de cette vision du monde dans l'interprétation des événements historiques de la fin du XIX et du début du Xx ${ }^{e}$ siècle. Cette négligence serait aussi paresse, vu la difficulté pour des intellectuels bourgeois, rationalistes et matérialistes, à entrer en sympathie avec un monde auquel ils ne comprennent rien et qu'ils prétendent pourtant juger dans son appréhension d'événements aussi importants que la révolution de $1905 \mathrm{ou}$ la Première Guerre mondiale. Au demeurant, cette attitude envers la Russie paysanne ne serait qu'un exemple de la vision des élites intellectuelles à l'égard des sociétés «traditionnelles » en général.

2 Contre cette conception qu'il critique, Leonid Heretz affirme la vitalité de la vision du monde traditionnelle portée par la paysannerie jusque dans cette période charnière de l'histoire russe, marquée par les phénomènes d'industrialisation et d'urbanisation qui ont orienté l'attention des historiens vers l'étude des transformations sociales et des changements culturels. Le monde des paysans n'était certes pas immobile, mais leur façon de voir ce mouvement restait fortement emprunt de la mentalité traditionnelle. C'est justement cela que l'auteur veut montrer dans cette étude, en insistant sur les conséquences des cette " persistance de la tradition » pour comprendre la Russie tsariste avant la révolution de 1917. 
3 Pour ce faire, il est absolument nécessaire, dit l'auteur, de reconnaître « le fait de la religiosité populaire » et la dimension culturelle fondamentale de la religion dans la vie russe $^{1}$. En posant la distinction centrale entre tradition et modernité et en se réclamant des concepts et de la méthode de Max Weber, l'auteur dénonce une certaine façon d'« objectiver» la religiosité de la paysannerie russe pour mieux la dévaloriser. On ne reconnaît en général aux paysans de l'époque qu'un vernis de religiosité chrétienne, pour voir en eux, soit de parfaits matérialistes acquis, malgré les apparences, à la logique de la rationalité économique, soit au contraire des païens dont l'appartenance à l'orthodoxie aurait été seulement superficielle. Ces deux interprétations reviennent, selon l'auteur, à nier la façon dont les paysans se percevaient eux-mêmes, c'est-à-dire comme des chrétiens orthodoxes. Or c'est cela qui constituerait la seule chose digne d'être étudiée, et que l'auteur se fait fort de présenter dans son livre.

Celui-ci est organisé en neuf chapitres, qui se répartissent en deux parties (invisibles dans la structure et simplement annoncées en passant dans l'introduction). Ces deux sections, de longueur inégale, répondent à deux questions différentes et correspondent aussi à deux méthodes différentes d'investigation historique. La première, constituée des trois premiers chapitres, consiste à définir ce qu'est la vision traditionnelle du monde portée par la paysannerie russe et à dire comment elle s'est formée. Cette partie, en réalité, repose sur une réinterprétation d'éléments antérieurs à la période sur laquelle l'auteur a l'ambition de renouveler notre regard. Cette étape préliminaire lui permet ensuite, dans la seconde partie, de traiter la question de la persistance de la tradition. Il s'agit là, en six chapitres, d'étudier comment la paysannerie, avec sa mentalité traditionnelle, comprenait l'histoire - ce que l'auteur appelle l'« eschatologie populaire» (chapitre 4) et comment elle interprétait un certain nombre d'événements historiques majeurs de la période : l'assassinat du tsar Alexandre II, la famine et l'épidémie de choléra de 1891-1892, la guerre russo-japonaise, la révolution de 1905 et enfin la Grande Guerre (chapitres 5 à 9).

5 Pourtant l'idée qu'il est nécessaire de révéler la persistance de la tradition chez les paysans, pour rompre avec les conceptions des observateurs russes de l'époque, ne laisse pas de surprendre, tant cet ancrage du monde paysan dans la tradition fut débattu au sein de l'intelligentsia ${ }^{2}$. La différence tient bien entendu à ce que la vision de l'auteur valorise la force et la stabilité de la tradition paysanne, là où nombre d'observateurs de l'époque n'y voyaient qu'archaïsme. Le sujet n'aurait pas beaucoup d'intérêt, s'il ne s'agissait que d'une pétition de principe. Mais l'auteur apporte un grand nombre d'exemples, présentés de façon vivante et tirés de la littérature ethnographique de l'époque, de la presse, voire de textes publiés par ceux que l'auteur identifie comme des porteurs particuliers de la mentalité traditionnelle, tels les vieux-croyants.

6 L'utilisation de ces différents exemples pose deux problèmes principaux. L'auteur mentionne brièvement dans son introduction le premier. Celui-ci touche à la nature des sources auxquelles il puise généralement pour bâtir sa démonstration, à savoir tous ces «instantanés» de vie paysanne recueillis par les ethnographes de l'époque, par des journalistes ou des publicistes. Certains chapitres du livre commencent par une présentation des sources et une mise en perspective de leur utilisation. Mais la méthode de l'auteur reste très généralisante, peu attentive aux divers contextes, sociaux et géographiques, de production des documents qu'il utilise, au motif que les idées priment et que la mentalité traditionnelle qu'il décrit concernait de toute façon l'immense majorité de la population russe. Ce choix permet à l'auteur de donner sur un grand nombre de thèmes des aperçus bien écrits et souvent intéressants, mais qui ne peuvent 
prétendre à une grande originalité dans le tableau des connaissances historiques sur cette période. Pourtant, c'est bien cette originalité que l'auteur revendique, pour comparer son travail à toute la production des chercheurs occidentaux qu'il critique uniformément pour leur surdité devant les réalités paysannes et pour leur façon de ne voir que les changements qui affectaient ces dernières. Mais que des historiens aient cherché à étudier ces changements ne signifie nullement qu'ils étaient sourds à la persistance des mœurs et des idées traditionnelles. D'ailleurs, dans l'étude de tel ou tel thème particulier, comme la révolution de 1905, l'auteur reconnaît bien que le rapport entre changement et persistance de la tradition doit, plus modestement, être étudié à partir de questions plus précises et mieux contextualisées (p.157-158), et qu'il ne s'agit pas de nier le changement (p. 183-189). Le dernier chapitre, sur la Grande Guerre, est encore plus net sur ce point, et permet de justifier le titre du livre.

7 Le second problème est lié à l'articulation entre les deux parties du livre. La première partie propose une description générale plutôt séduisante de la vision du monde traditionnelle. Cette description est partiellement a-chronologique, mais attentive aux diverses influences qui ont façonné la vision traditionnelle, dont la sensibilité religieuse est le fin mot. L'auteur montre les différentes sources auxquelles allait puiser cette sensibilité : non seulement les enseignements de l'Église orthodoxe officielle, mais aussi les sources apocryphes, qui se combinaient aux premières pour former la façon de voir du peuple. Leonid Heretz fait aussi une place particulière, dans les chapitres 2 et 3, à la part prise par les dissidents religieux qu'étaient les vieux-croyants et les membres de diverses sectes dans l'élaboration de la réponse traditionaliste aux forces «modernisatrices» dans l'histoire russe depuis le XVII siècle. Les éléments présentés de façon synthétique sont d'un grand intérêt, mais l'auteur postule plus qu'il ne démontre l'influence de ces idées sur le reste de la paysannerie (p. 59), c'est-à-dire sur l'immense majorité de la population, porteuse de cette fameuse vision traditionnelle du monde ${ }^{3}$. Les voies de cette influence ne sont pas explorées dans le reste du livre. Au contraire, dans la seconde partie, le chapitre fondamental sur « l'eschatologie populaire », dans les rares mentions qu'il fait de la vieille foi, insiste plutôt sur ses particularités par comparaison avec la mentalité traditionnelle dominante (p. 104, 107, 112). Aussi, l'idée de l'auteur qu'il est possible de reconstruire une notion unifiée de la mentalité russe traditionnelle se heurte à la variété des courants, des tendances et des influences qu'il essaie bien de présenter, mais qu'il ne réussit pas toujours à lier les uns aux autres.

8 L'ouvrage de Leonid Heretz suscite donc une interrogation intéressante. C'est celle de savoir s'il suffit de partir d'une critique globale (et sans nuance) des travaux historiques antérieurs pour produire une recherche novatrice. L'auteur a eu tendance à s'enfermer dans son combat pour réhabiliter la tradition dans le regard universitaire sur la Russie d'ancien régime. Mais il a un peu trop délaissé, nous semble-t-il, les pistes qu'il avait luimême repérées au début de son livre et grâce auxquelles il aurait pu mieux faire comprendre les tensions internes à cette vision du monde traditionnelle si chère à son coeur. 


\section{NOTES}

1. À cet égard, l'auteur apporte sa contribution à un courant de recherches en expansion depuis quelques années, notamment avec les livres de Vera Shevzov, Russian Orthodoxy on the Eve of Revolution, Oxford : Oxford University Press, 2004 et de Chris J. Chulos, Converging Worlds : Religion and Community in Peasant Russia, 1861-1917, DeKalb : Northern Illinois University Press, 2003. Mais la bibliographie de l'ouvrage n'est pas à ce jour sur ce point.

2. On s'étonne aussi qu'il n'y ait aucune référence au livre fondamental sur ces perceptions du monde paysan par les élites cultivées de l'époque: Cathy A. Frierson, Peasant Icons: Representations of Rural People in Late Nineteenth-Century Russia, Oxford: Oxford University Press, 1993. Cet ouvrage montre d'ailleurs que les regards sur les paysans étaient beaucoup plus variés et divergents que ne le laisse entendre ici l'auteur.

3. En fait, l'auteur ne place pas sur le même plan son étude des vieux-croyants et celle des «sectaires ». L'étude des vieux-croyants postule l'influence de ce courant sur la paysannerie restée orthodoxe ; au contraire, l'étude des mouvements sectaires présente plutôt ces derniers comme un révélateur des idées traditionnelles préexistant dans le peuple orthodoxe. 